\title{
Families of classical subgroup separable superintegrable systems
}

\author{
E. G. Kalnins \\ Department of Mathematics, University of Waikato, \\ Hamilton, New Zealand \\ J. M. Kress \\ School of Mathematics and Statistics, University if New South Wales, \\ Sydney, Australia \\ W. Miller, Jr. \\ School of Mathematics, University of Minnesota, \\ Minneapolis, Minnesota, U. S. A.
}

October 30, 2018

\begin{abstract}
We describe a method for determining a complete set of integrals for a classical Hamiltonian that separates in orthogonal subgroup coordinates. As examples, we use it to determine complete sets of integrals, polynomial in the momenta, for some families of generalized oscillator and Kepler-Coulomb systems, hence demonstrating their superintegrability. The latter generalizes recent results of Verrier and Evans, and Rodríguez, Tempesta, and Winternitz. Another example is given of a superintegrable system on a non-conformally flat space.
\end{abstract}

An $n$-dimensional classical superintegrable system is an integrable Hamiltonian system that not only possesses $n$ mutually commuting integrals, but in addition, the Hamiltonian Poissoncommutes with $2 n-1$ functions on the phase space that are globally defined and polynomial in the momenta. This notion can be extended to define a quantum superintegrable system as a quantum Hamiltonian which is one of a set of $n$ independent mutually commuting differential operators that commutes with a set of $2 n-1$ independent differential operators of finite order.

Such systems have been studied because of their associated algebras that allow direct calculation of spectral decompositions and their connections with separation of variables and special 
function theory. There has also been great interest in quantum superintegrable systems because it has been conjectured that they coincide with quasi-exactly solvable systems [1], [2]).

Recently, an infinite family of exactly solvable quantum mechanical systems was studied by Tremblay, Turbiner and Winternitz (TTW) [3]. The proposition that every member of this family of systems is superintegrable, while not proven, is supported by the fact that their classical counterparts have been shown to be superintegrable [4].

In this paper the methods of [5] and [4] are used to demonstrate that some natural $n$-dimensional generalizations of the TTW class of classical systems are superintegrable, that is, possess $2 n-1$ constants that are polynomial in the momenta. All superintegrable systems previously known to us exist on conformally flat spaces. However, the underlying metrics of the new systems presented here are not necessarily conformally flat, as we show explicitly.

In the first section we discuss a general method for determining additional constants for a Hamiltonian written in a particular type of separable coordinates. This method is then applied to a generalization of the family of singular oscillator systems given in [3] and to a family of generalized extended Kepler-Coulomb systems. We conclude with an example of a nonconformally flat superintegrable system in four dimensions. Our methods clearly extend to a wide variety of $n$-dimensional systems. Finding the quantum analogs of these systems is a nontrivial problem.

\section{Superintegrability of subgroup separable Hamiltoni- ans}

Consider the $n$ functions

$$
\begin{aligned}
L_{i} & =p_{i}^{2}+V_{i}\left(q_{i}\right)+f_{i}\left(q_{i}\right) L_{i+1}, \quad i=1, \ldots, n-1, \\
L_{n} & =p_{n}^{2}+V_{n}\left(q_{n}\right),
\end{aligned}
$$

on a $2 n$-dimensional phase space with position coordinates $q_{i}$ and conjugate momenta $p_{i}, i=$ $1, \ldots, n$. Each $L_{i}$ is a function of $q_{i}, \ldots, q_{n}, p_{i}, \ldots, p_{n}$ and the Hamiltonian $H=L_{1}$ is clearly separable in the coordinates $q_{i}$. Furthermore, the set $\left\{L_{1}, L_{2}, \ldots, L_{n}\right\}$ is in involution, that is $\left\{L_{i}, L_{j}\right\}=0$ for all $i, j=1, \ldots, n$, where $\{$,$\} is the usual Poisson bracket,$

$$
\{F, G\}=\sum_{i=1}^{n} \frac{\partial F}{\partial p_{i}} \frac{\partial G}{\partial q_{i}}-\frac{\partial F}{\partial q_{i}} \frac{\partial G}{\partial p_{i}}
$$

We can find $n-1$ additional independent functions commuting with $H$ by first finding, for each $i=1, \ldots, n-1$, a pair of functions $M_{i}\left(q_{i}, L_{1}, L_{2}, \ldots, L_{n}\right)$ and $N_{i}\left(q_{i+1}, L_{1}, L_{2}, \ldots, L_{n}\right)$ satisfying

$$
\left\{H, M_{i}\right\}=\prod_{k=1}^{i} f_{k}\left(q_{k}\right) \quad \text { and } \quad\left\{H, N_{i}\right\}=\prod_{k=1}^{i} f_{k}\left(q_{k}\right)
$$


Then $\left\{H, N_{i}-M_{i}\right\}=0$ and hence

$$
L_{i}^{\prime}=N_{i}-M_{i}
$$

is in involution with $H$. These additional functions need not be globally defined nor polynomial in the momenta,. However, for the examples given in later sections each $L_{i}^{\prime}$ gives rise to an additional constant polynomial in the momenta.

Now,

$$
\left\{H, M_{i}\right\}=2 p_{i} \frac{\partial M_{i}}{\partial q_{i}} \prod_{k=1}^{i-1} f_{k}\left(q_{k}\right) \quad \text { and } \quad\left\{H, N_{i}\right\}=2 p_{i+1} \frac{\partial N_{i}}{\partial q_{i+1}} \prod_{k=1}^{i} f_{k}\left(q_{k}\right)
$$

and so equations (2) become

$$
2 p_{i} \frac{\partial M_{i}}{\partial q_{i}}=f_{i}\left(q_{i}\right) \quad \text { and } \quad 2 p_{i+1} \frac{\partial N_{i}}{\partial q_{i+1}}=1
$$

To solve these equations we note that using (1) we can write $p_{i}$ in terms of $L_{i}$ and then treat $L_{i}$ and $L_{i+1}$ as constants. Hence we find

$$
\begin{aligned}
M_{i} & =\int \frac{f_{i}\left(q_{i}\right) d q_{i}}{2 \sqrt{L_{i}-V_{i}\left(q_{i}\right)-f_{i}\left(q_{i}\right) L_{i+1}}} \\
N_{i} & =\int \frac{d q_{i}}{2 \sqrt{L_{i+1}-V_{i+1}\left(q_{i+1}\right)-f_{i+1}\left(q_{i+1}\right) L_{i+2}}} .
\end{aligned}
$$

To write these in a consistent form for $i=1, \ldots, n-1$ we take $L_{n+1}=0$.

We also need to check that set $\left\{L_{1}, \ldots, L_{n}, L_{1}^{\prime}, \ldots, L_{n-1}^{\prime}\right\}$ is indeed functionally independent. First we note that

$$
\left\{L_{i}, M_{j}\right\}=\left\{\begin{array}{ll}
0 & i>j \\
\prod_{k=i}^{j} f_{k}\left(q_{k}\right) & i \leq j
\end{array} \quad\left\{L_{i}, N_{j}\right\}= \begin{cases}0 & i>j+1 \\
1 & i=j+1 \\
\prod_{k=i}^{j} f_{k}\left(q_{k}\right) & i<j+1\end{cases}\right.
$$

and so

$$
\left\{L_{i}, L_{i-1}^{\prime}\right\}=1 \quad \text { for } i>1 \quad \text { and } \quad\left\{L_{i}, L_{j}^{\prime}\right\}=0 \quad \text { for } j \neq i-1 .
$$

If we assume that there exists a function $F$ such that

$$
F\left(L_{1}, \ldots, L_{n}, L_{1}^{\prime}, \ldots, L_{n-1}^{\prime}\right)=0
$$

then for each $j=2, \ldots, n$,

$$
0=\left\{L_{j}, F\right\}=\sum_{k=1}^{n}\left\{L_{j}, L_{k}\right\} \frac{\partial F}{\partial L_{k}}+\sum_{k=1}^{n-1}\left\{L_{j}, L_{k}^{\prime}\right\} \frac{\partial F}{\partial L_{k}^{\prime}}=\left\{L_{j}, L_{j-1}^{\prime}\right\} \frac{\partial F}{\partial L_{j-1}^{\prime}}=\frac{\partial F}{\partial L_{j-1}^{\prime}} .
$$


So $F$ can not depend on any of the $L_{i}^{\prime}$ and equation (5) must be a functional relationship between the $L_{1}, \ldots, L_{n}$ alone which are are clearly functionally independent. Hence the set $\left\{L_{1}, \ldots, L_{n}, L_{1}^{\prime}, \ldots, L_{n-1}^{\prime}\right\}$ must be functionally independent.

Note that if we were to find an additional function $L_{n}^{\prime}$ such that $\left\{H, L_{n}^{\prime}\right\}=1$, (which we can [5]) then the set $\left\{L_{1}, \ldots, L_{n}, L_{1}^{\prime}, \ldots, L_{n}^{\prime}\right\}$ would constitute a set of action-angle variables for the system [6].

\section{A three-dimensional example}

The details of the calculation for the TTW systems (defined on $E(2, \mathbb{C})$ ) have already been given in [4], so here we first consider the Hamiltonian on $E(3, \mathbb{C})$

$$
H=p_{x}^{2}+p_{y}^{2}+p_{z}^{2}+\alpha\left(x^{2}+y^{2}+z^{2}\right)+\frac{\beta_{1}}{z^{2}}+\frac{\beta_{2}}{x^{2}}+\frac{\beta_{3}}{y^{2}}
$$

which in polar coordinates is

$$
H=p_{r}^{2}+\frac{p_{\theta_{1}}^{2}}{r^{2}}+\frac{p_{\theta_{2}}^{2}}{r^{2} \sin ^{2} \theta_{1}}+\alpha r^{2}+\frac{\beta_{1}}{r^{2} \cos ^{2} \theta_{1}}+\frac{\beta_{2}}{r^{2} \sin ^{2} \theta_{1} \cos ^{2} \theta_{2}}+\frac{\beta_{3}}{r^{2} \sin ^{2} \theta_{1} \sin ^{2} \theta_{2}}
$$

and has the form discussed in section 1. We modify this so that

$$
H=p_{r}^{2}+\frac{p_{\theta_{1}}^{2}}{r^{2}}+\frac{p_{\theta_{2}}^{2}}{r^{2} \sin ^{2}\left(k_{1} \theta_{1}\right)}+\alpha r^{2}+\frac{\beta_{1}}{r^{2} \cos ^{2}\left(k_{1} \theta_{1}\right)} \cdot+\frac{\beta_{2}}{r^{2} \sin ^{2}\left(k_{1} \theta_{1}\right) \cos ^{2}\left(k_{2} \theta_{2}\right)}+\frac{\beta_{3}}{r^{2} \sin ^{2}\left(k_{1} \theta_{1}\right) \sin ^{2}\left(k_{2} \theta_{2}\right)}
$$

with $k_{1}$ and $k_{2}$ two positive rational parameters. Note that when $k_{1} \neq 1$, this is no longer a natural Hamiltonian on a flat space, however, the Cotton-York tensor of the corresponding metric vanishes identically for all $k_{1}$ and hence the underlying space is conformally flat.

The Hamilton-Jacobi equation separates due to the second order constants

$$
L_{3}=p_{\theta_{2}}^{2}+\frac{\beta_{2}}{\cos ^{2}\left(k_{2} \theta_{2}\right)}+\frac{\beta_{3}}{\sin ^{2}\left(k_{2} \theta_{2}\right)}
$$

and

$$
L_{2}=p_{\theta_{1}}^{2}+\frac{\beta_{1}}{\cos ^{2}\left(k_{1} \theta_{1}\right)}+\frac{L_{3}}{\sin ^{2}\left(k_{1} \theta_{1}\right)} .
$$

and the Hamiltonian can be written as

$$
H=L_{1}=p_{r}^{2}+\alpha r^{2}+\frac{L_{2}}{r^{2}}
$$

Now we look for additional constants by finding $M_{1}\left(r, H, L_{2}, L_{3}\right)$ and $N_{1}\left(\theta_{1}, H, L_{2}, L_{3}\right)$ such that

$$
\left\{H, M_{1}\right\}=\frac{1}{r^{2}} \quad \text { and } \quad\left\{H, N_{1}\right\}=\frac{1}{r^{2}}
$$


and $M_{2}\left(\theta_{1}, H, L_{2}, L_{3}\right)$ and $N_{2}\left(\theta_{2}, H, L_{2}, L_{3}\right)$ satisfying

$$
\left\{H, M_{2}\right\}=\frac{1}{r^{2} \sin ^{2}\left(k_{1} \theta_{1}\right)} \quad \text { and } \quad\left\{H, N_{2}\right\}=\frac{1}{r^{2} \sin ^{2}\left(k_{1} \theta_{1}\right)} .
$$

Then $L_{1}^{\prime}=N_{1}-M_{1}$ and $L_{2}^{\prime}=N_{2}-M_{2}$ will be constants of the motion. To compute these we need to calculate the following 4 integrals,

$$
\begin{aligned}
& M_{1}=\int \frac{d r}{2 r^{2} \sqrt{H-\alpha r^{2}-\frac{L_{2}}{r^{2}}}} \\
& N_{1}=\int \frac{d \theta_{1}}{2 \sqrt{L_{2}-\frac{\beta_{1}}{\cos ^{2}\left(k_{1} \theta_{1}\right)}-\frac{L_{3}}{\sin ^{2}\left(k_{1} \theta_{1}\right)}}} \\
& M_{2}=\int \frac{d \theta_{1}}{2 \sin ^{2}\left(k_{1} \theta_{1}\right) \sqrt{L_{2}-\frac{\beta_{1}}{\cos ^{2}\left(k_{1} \theta_{1}\right)}-\frac{L_{3}}{\sin ^{2}\left(k_{1} \theta_{1}\right)}}} \\
& N_{2}=\int \frac{d \theta_{2}}{2 \sqrt{L_{3}-\frac{\beta_{2}}{\cos ^{2}\left(k_{2} \theta_{2}\right)}-\frac{\beta_{3}}{\sin ^{2}\left(k_{2} \theta_{2}\right)}}}
\end{aligned}
$$

in which $H=L_{1}, L_{2}$ and $L_{3}$ are treated as constants. We find

$$
M_{1}=\frac{\mathcal{B}_{1}}{4 \sqrt{-L_{2}}}, \quad N_{1}=\frac{\mathcal{A}_{1}}{4 k_{1} \sqrt{-L_{2}}}, \quad M_{2}=\frac{\mathcal{B}_{2}}{4 k_{1} \sqrt{-L_{3}}} \quad \text { and } \quad N_{2}=\frac{\mathcal{A}_{2}}{4 k_{2} \sqrt{-L_{3}}}
$$

where

$$
\begin{aligned}
\sinh \mathcal{B}_{1} & =i \frac{H-\frac{2 L_{2}}{r^{2}}}{\sqrt{H^{2}-4 \alpha L_{2}}} & \cosh \mathcal{B}_{1} & =-\frac{2 \sqrt{L_{2}} p_{r}}{r \sqrt{H^{2}-4 \alpha L_{2}}} \\
\sinh \mathcal{A}_{1} & =i \frac{L_{2} \cos \left(2 k_{1} \theta_{1}\right)+L_{3}-\beta_{1}}{\sqrt{\left(\beta_{1}-L_{2}-L_{3}\right)^{2}-4 L_{2} L_{3}}} & \cosh \mathcal{A}_{1} & =\frac{\sqrt{L_{2}} \sin \left(2 k_{1} \theta_{1}\right) p_{\theta_{1}}}{\sqrt{\left(\beta_{1}-L_{2}-L_{3}\right)^{2}-4 L_{2} L_{3}}} \\
\sinh \mathcal{B}_{2} & =\frac{2 L_{3} \operatorname{cosec}^{2}\left(k_{1} \theta_{1}\right)+\beta_{1}-L_{2}-L_{3}}{\sqrt{4 \beta_{1} L_{2}-\left(L_{3}-L_{2}-\beta_{1}\right)^{2}}} & \cosh \mathcal{B}_{2} & =-\frac{2 i \sqrt{L_{3}} \cot \left(k_{1} \theta_{1}\right) p_{\theta_{1}}}{\sqrt{4 \beta_{1} L_{2}-\left(L_{3}-L_{2}-\beta_{1}\right)^{2}}} \\
\sinh \mathcal{A}_{2} & =i \frac{L_{3} \cos \left(2 k_{2} \theta_{2}\right)+\beta_{3}-\beta_{2}}{\sqrt{\left(\beta_{2}-\beta_{3}-L_{3}\right)^{2}-4 \beta_{3} L_{3}}} & \cosh \mathcal{A}_{2} & =\frac{\sqrt{L_{3}} \sin \left(2 k_{2} \theta_{2}\right) p_{\theta_{2}}}{\sqrt{\left(\beta_{2}-\beta_{3}-L_{3}\right)^{2}-4 \beta_{3} L_{3}}} .
\end{aligned}
$$

To ensure that we can find polynomial constants, we have chosen $k_{1}$ and $k_{2}$ to be rational and so we can take

$$
k_{1}=\frac{p_{1}}{q_{1}} \quad \text { and } \quad k_{2}=\frac{p_{2}}{q_{2}} \quad \text { with } \quad p_{1}, q_{1}, p_{2}, q_{2} \in \mathbb{Z}^{+} \quad \text { and } \quad \operatorname{gcd}\left(p_{1}, q_{1}\right)=\operatorname{gcd}\left(p_{2}, q_{2}\right)=1,
$$

and then

$$
\sinh \left(4 p_{1} \sqrt{-L_{2}}\left(N_{1}-M_{1}\right)\right) \quad \text { and } \quad \sinh \left(4 p_{1} p_{2} \sqrt{-L_{3}}\left(N_{2}-M_{2}\right)\right)
$$


are constants of the motion having the form of a polynomial in the momenta divided by a function of $H, L_{2}$, and $L_{3}$. This follows from the elementary relations [4]

$$
\begin{aligned}
(\cosh x \pm \sinh x)^{n}= & \cosh n x \pm \sinh n x, \cosh (x+y)=\cosh x \cosh y+\sinh x \sinh y \\
& \sinh (x+y)=\cosh x \sinh y+\sinh x \cosh y
\end{aligned}
$$

In particular,

$$
\begin{gathered}
\cosh n x=\sum_{j=0}^{[n / 2]}\left(\begin{array}{c}
n \\
2 j
\end{array}\right) \sinh ^{2 j} x \cosh ^{n-2 j} x, \\
\sinh n x=\sinh x \sum_{j=1}^{[(n=1) / 2]}\left(\begin{array}{c}
n \\
2 j-1
\end{array}\right) \sinh ^{2 j-2} x \cosh ^{n-2 j-1} x .
\end{gathered}
$$

From these we can find $L_{1}^{\prime \prime}$ and $L_{2}^{\prime \prime}$ such that $\left\{H, L_{2}, L_{3}, L_{1}^{\prime \prime}, L_{2}^{\prime \prime}\right\}$ is a set of functionally independent constants of the motion polynomial in the momenta.

Note these polynomial constants are not necessarily of minimal degree. Indeed, if we set $k_{1}=$ $k_{2}=1$ and recover the usual Smorodinski-Winternitz potential, we find additional constants $L_{1}^{\prime \prime}$ and $L_{2}^{\prime \prime}$ that are cubic in the momenta, whereas it is well known that there exist additional quadratic constants.

\section{Extended Kepler-Coulomb system}

The same procedure works with the harmonic oscillator term $\alpha r^{2}$ in (6) replaced by a KeplerCoulomb term $\alpha / r$, that is,

$$
H=p_{r}^{2}+\frac{p_{\theta_{1}}^{2}}{r^{2}}+\frac{p_{\theta_{2}}^{2}}{r^{2} \sin ^{2}\left(k_{1} \theta_{1}\right)}+\frac{\alpha}{r}+\frac{\beta_{1}}{r^{2} \cos ^{2}\left(k_{1} \theta_{1}\right)} \cdot+\frac{\beta_{2}}{r^{2} \sin ^{2}\left(k_{1} \theta_{1}\right) \cos ^{2}\left(k_{2} \theta_{2}\right)}+\frac{\beta_{3}}{r^{2} \sin ^{2}\left(k_{1} \theta_{1}\right) \sin ^{2}\left(k_{2} \theta_{2}\right)}
$$

This system is superintegrable for rational $k_{1}$ and $k_{2}$. All calculations are the same as for the singular oscillator except

$$
M_{1}=\int \frac{d r}{2 r^{2} \sqrt{H-\frac{\alpha}{r}-\frac{L_{2}}{r^{2}}}}
$$

and

$$
M_{1}=\frac{1}{2 \sqrt{-L_{2}}} \sinh ^{-1}\left(i \frac{\alpha+\frac{2 L_{2}}{r}}{\sqrt{\alpha^{2}+4 H L_{2}}}\right)
$$

or

$$
M_{1}=\frac{\mathcal{B}_{1}}{2 \sqrt{-L_{2}}} \quad \text { where } \quad \sinh \mathcal{B}_{1}=i \frac{\alpha+\frac{2 L_{2}}{r}}{\sqrt{\alpha^{2}+4 H L_{2}}} \quad \text { and } \quad \cosh \mathcal{B}_{1}=\frac{2 \sqrt{L_{2}} p_{r}}{\sqrt{\alpha^{2}+4 H L_{2}}}
$$


Verrier and Evans [7], and Rodríguez, Tempesta, and Winternitz [8], separately, considered the $k_{1}=k_{2}=1$ case of this family of systems and found it to be superintegrable with 4 second order and one fourth order constant. The method presented here leads to, in addition to the 3 second order constants $H, L_{2}$ and $L_{3}$, a third and a fourth order constant.

\section{A non-flat higher-dimensional example}

The three-dimensional examples above can readily be extended to $n$ dimensions to give families of $n$-dimensional superintegrable Hamiltonians, each with $2 n-1$ functionally independent polynomial constants of the momenta. In 4-dimensions, by calculating the Weyl tensor, it can be seen that the corresponding metric is conformally flat if and only if $k_{1}=k_{2}$. Hence we can generate examples of superintegrable systems on non-conformally flat spaces.

As an example consider the natural generalization of the example above to 4 dimensions with $k_{1}=2$ and $k_{2}=k_{3}=1$. That is,

$$
\begin{aligned}
L_{4} & =p_{\theta_{3}}^{2}+\frac{\beta_{3}}{\cos ^{2}\left(\theta_{3}\right)}+\frac{\beta_{4}}{\sin ^{2}\left(\theta_{3}\right)} \\
L_{3} & =p_{\theta_{2}}^{2}+\frac{\beta_{2}}{\cos ^{2}\left(\theta_{2}\right)}+\frac{L_{4}}{\sin ^{2}\left(\theta_{2}\right)} \\
L_{2} & =p_{\theta_{1}}^{2}+\frac{\beta_{1}}{\cos ^{2}\left(2 \theta_{1}\right)}+\frac{L_{3}}{\sin ^{2}\left(2 \theta_{1}\right)} \\
H=L_{1} & =p_{r}^{2}+\alpha r^{2}+\frac{L_{2}}{r^{2}} .
\end{aligned}
$$

As in the previous example, we calculate $M_{1}\left(r, H, L_{2}, L_{3}, L_{4}\right), N_{1}\left(\theta_{1}, H, L_{2}, L_{3}, L_{4}\right), M_{2}\left(\theta_{1}, H, L_{2}, L_{3}, L_{4}\right)$, $N_{2}\left(\theta_{2}, H, L_{2}, L_{3}, L_{4}\right), M_{3}\left(\theta_{2}, H, L_{2}, L_{3}, L_{4}\right)$ and $N_{3}\left(\theta_{3}, H, L_{2}, L_{3}, L_{4}\right)$ using (44) to give very similar expressions. We can form polynomial constants from $L_{i}^{\prime}=N_{i}-M_{i}$ for $i=1,2,3$.

$$
\begin{aligned}
\sinh \left(8 \sqrt{-L_{2}}\left(N_{1}-M_{1}\right)\right)= & \sinh \left(\mathcal{A}_{1}-2 \mathcal{B}_{1}\right) \\
= & -2 \cosh \mathcal{A}_{1} \sinh \mathcal{B}_{1} \cosh \mathcal{B}_{1}+2 \sinh \mathcal{A}_{1} \cosh ^{2} \mathcal{B}_{1}-\sinh \mathcal{A}_{1} \\
= & \frac{4 i L_{2}\left(\left(H-\frac{2 L_{2}}{r^{2}}\right) \frac{\sin \left(4 \theta_{1}\right)}{r} p_{\theta_{1}} p_{r}+\frac{2\left(L_{2} \cos \left(4 \theta_{1}\right)+L_{3}-\beta_{1}\right)}{r^{2}} p_{r}^{2}\right)}{\left(H^{2}-4 \alpha L_{2}\right) \sqrt{\left(\beta_{1}-L_{2}-L_{3}\right)^{2}-4 L_{2} L_{3}}} \\
& -i \frac{\left(H^{2}-4 \alpha L_{2}\right)\left(L_{2} \cos \left(4 \theta_{1}\right)+L_{3}-\beta_{1}\right)}{\left(H^{2}-4 \alpha L_{2}\right) \sqrt{\left(\beta_{1}-L_{2}-L_{3}\right)^{2}-4 L_{2} L_{3}}} .
\end{aligned}
$$

The denominator of this expression is a constant of the motion and hence so is the numerator which is clearly a polynomial in the momenta. We also can find a lower degree constant $L_{1}^{\prime \prime}$ by 
noting that

$$
\sinh \left(8 \sqrt{-L_{2}}\left(N_{1}-M_{1}\right)\right)=\frac{4 i L_{2} L_{1}^{\prime \prime}-i H^{2}\left(L_{3}-\beta_{1}\right)}{\left(H^{2}-4 \alpha L_{2}\right) \sqrt{\left(\beta_{1}-L_{2}-L_{3}\right)^{2}-4 L_{2} L_{3}}}
$$

where

$$
L_{1}^{\prime \prime}=\left(H-\frac{2 L_{2}}{r^{2}}\right) \frac{\sin \left(2 \theta_{1}\right)}{r} p_{\theta_{1}} p_{r}+\frac{2\left(L_{2} \cos \left(2 \theta_{1}\right)+L_{3}-\beta_{1}\right)}{r^{2}} p_{r}^{2}-\frac{1}{4}\left(H^{2}-\alpha L_{2}\right) \cos \left(4 \theta_{1}\right)
$$

is a constant quartic in he momenta.

Similarly,

$$
\begin{aligned}
\sinh \left(8 \sqrt{-L_{3}}\left(N_{2}-M_{2}\right)\right) \\
=\sinh \left(2 \mathcal{A}_{2}-\mathcal{B}_{2}\right) \\
=-2 \cosh ^{2} \mathcal{A}_{2} \sinh \mathcal{B}_{2}+2 \sinh \mathcal{A}_{2} \cosh \mathcal{A}_{2} \cosh \mathcal{B}_{2}+\sinh \mathcal{B}_{2} \\
=L_{3} \frac{2\left(L_{3} \cos \left(2 \theta_{2}\right)+L_{4}-\beta_{2}\right) \cot \left(2 \theta_{1}\right) \sin \left(2 \theta_{2}\right) p_{\theta_{1}} p_{\theta_{2}}-\sin ^{2}\left(2 \theta_{2}\right)\left(2 L_{3} \operatorname{cosec}^{2}\left(2 \theta_{1}\right)+\beta_{1}-L_{2}-L_{3}\right) p_{\theta_{2}}^{2}}{\left(\left(\beta_{2}-L_{3}-L_{4}\right)^{2}-4 L_{3} L_{4}\right) \sqrt{4 \beta_{1} L_{2}-\left(L_{3}-L_{2}-\beta_{1}\right)^{2}}} \\
\quad+\frac{\left(\left(\beta_{2}-L_{3}-L_{4}\right)^{2}-4 L_{3} L_{4}\right)\left(2 L_{3} \operatorname{cosec}^{2}\left(2 \theta_{1}\right)+\beta_{1}-L_{2}-L_{3}\right)}{\left(\left(\beta_{2}-L_{3}-L_{4}\right)^{2}-4 L_{3} L_{4}\right) \sqrt{4 \beta_{1} L_{2}-\left(L_{3}-L_{2}-\beta_{1}\right)^{2}}} .
\end{aligned}
$$

Hence

$$
\begin{gathered}
L_{2}^{\prime \prime}=2\left(L_{3} \cos \left(2 \theta_{2}\right)+L_{4}-\beta_{2}\right) \cot \left(2 \theta_{1}\right) \sin \left(2 \theta_{2}\right) p_{\theta_{1}} p_{\theta_{2}}+\left(\left(\beta_{2}-L_{3}-L_{4}\right)^{2}-4 L_{3} L_{4}\right) \operatorname{cosec}^{2}\left(2 \theta_{1}\right) \\
-\sin ^{2}\left(2 \theta_{2}\right)\left(2 L_{3} \operatorname{cosec}^{2}\left(2 \theta_{1}\right)+\beta_{1}-L_{2}-L_{3}\right) p_{\theta_{2}}^{2}
\end{gathered}
$$

is an additional constant that is quartic in the momenta, and

$$
\begin{aligned}
& \sinh \left(4 \sqrt{-L_{4}}\left(N_{3}-M_{3}\right)\right) \\
& =\sinh \left(\mathcal{A}_{3}-\mathcal{B}_{3}\right) \\
& =\sinh \mathcal{A}_{3} \cosh \mathcal{B}_{3}-\cosh \mathcal{A}_{3} \sinh \mathcal{B}_{3} \\
& =\frac{\sqrt{L_{4}}\left(2\left(L_{4} \cos \left(2 \theta_{3}\right)+\beta_{4}-\beta_{3}\right) \cot \left(\theta_{2}\right) p_{\theta_{2}}-\left(2 L_{4} \operatorname{cosec}^{2}\left(\theta_{2}\right)+\beta_{2}-L_{3}-L_{4}\right) \sin \left(2 \theta_{3}\right) p_{\theta_{3}}\right)}{\sqrt{4 \beta_{2} L_{3}-\left(L_{4}-L_{3}-\beta_{2}\right)^{2}} \sqrt{\left(\beta_{3}-\beta_{4}-L_{4}\right)^{2}-4 \beta_{4} L_{4}}} .
\end{aligned}
$$

Hence

$$
L_{3}^{\prime \prime}=2\left(L_{4} \cos \left(2 \theta_{3}\right)+\beta_{4}-\beta_{3}\right) \cot \left(\theta_{2}\right) p_{\theta_{2}}-\left(2 L_{4} \operatorname{cosec}^{2}\left(\theta_{2}\right)+\beta_{2}-L_{3}-L_{4}\right) \sin \left(2 \theta_{3}\right) p_{\theta_{3}}
$$

is an additional constant that is cubic in the momenta.

So we have demonstrated the superintegrability of one member of this family of superintegrable systems by giving explicit expressions for $2 n-1$ functionally independent polynomial constants. In this case, the underlying space is curved and not conformally flat as has been the case for previously known superintegrable systems.

It is clear from this example, that similar results will be obtained for both this family of generalized oscillators and the family of generalized Kepler-Coulomb systems for any rational choices of the $k_{1}, k_{2}, \ldots, k_{n-1}$ in $n$ dimensions. 


\section{References}

[1] P. Tempesta, A. Turbiner and P. Winternitz. Exact solvability of superintegrable systems J. Math. Phys. 42 (2001) 4248-57.

[2] E.G.Kalnins, W.Miller Jr., and G.S.Pogosyan. Exact and quasi-exact solvability of second order superintegrable quantum systems. I Euclidean space preliminaries. J. Math. Phys., 47, 033502, 2006.

[3] F. Tremblay, V. A. Turbiner and P. Winternitz. An infinite family of solvable and integrable quantum systems on a plane. J. Phys. A: Math. Theor. 42 (2009) 242001.

[4] E. G. Kalnins, W. Miller Jr., and G. S. Pogosyan. Superintegrability and higher order constants for classical and quantum systems. (submitted) arXiv:0912.2278v1 [math-ph] (2009)

[5] E. G. Kalnins, J. M. Kress, W. Miller Jr., and G. S. Pogosyan. Complete sets of invariants for dynamical systems that admit separation of variables. J. Math. Phys., 43, 3592-3609,2002.

[6] V. I. Arnold. Mathematical Methods of Classical Mechanics, (Springer, Berlin, 1989)

[7] P. E. Verrier and N. W. Evans. A new superintegrable Hamiltonian. J. Math. Phys. 49 (2008) 022902.

[8] M. A. Rodríguez, P. Tempesta, and P. Winternitz. Symmetry reduction and superintegrable Hamiltonian systems Journal of Physics: Conference Series 175 (2009) 012013. 